\title{
Psychological morbidities and positive psychological outcomes in people with traumatic spinal cord injury in Mainland China
}

\author{
Yanbo Wang ${ }^{1} \cdot$ Haixia Xie ${ }^{2} \cdot$ Xudong Zhao ${ }^{1}$
}

Received: 15 April 2017 / Revised: 14 November 2017 / Accepted: 14 November 2017 / Published online: 9 February 2018

(C) International Spinal Cord Society 2018

\begin{abstract}
Study design Cross-sectional survey.

Objectives To explore the prevalences of three psychological morbidities (posttraumatic stress disorder, anxiety, and depression) and two positive psychological outcomes (resilience and posttraumatic growth) in people with spinal cord injury (SCI). To examine the relationships between the five aforementioned variables and to determine the predictors of the three psychological morbidities.

Setting Shanghai Sunshine Rehabilitation Center, Mainland China.

Methods Participants included 300 adults with SCI in one rehabilitation center in Shanghai. Standardized self-report measures were used. Sociodemographic, injury, and psychological variables were assessed. Descriptive analyses were used to calculate the prevalences of five psychological outcome variables. Pearson correlation analyses were conducted to examine the relationships between the five psychological variables and regression analyses were conducted to determine the predictors of posttraumatic stress disorder (PTSD), anxiety, and depression.

Results Of the 300 respondents, 35\%, 29\%, and 27\% exceeded the clinical cutoff score for PTSD, anxiety, and depression, respectively. About 32\% reported good resilience, and 51\% reported moderate to high levels of posttraumatic growth (PTG). Three psychological morbidities showed positive correlations between each other while significant negative relationships with the resilience and PTG. Hierarchical regressions indicated that both the extent of environmental barriers and resilience were the significant predictors of PTSD, anxiety, and depression.

Conclusion High prevalences of psychological morbidities were found in the SCI population in Mainland China. They should be identified and intervened early in the rehabilitation process. Some positive psychological techniques that focus on increasing resilience and promoting PTG would likely be beneficial for the SCI population.
\end{abstract}

\section{Introduction}

Traumatic spinal cord injury (SCI) is often sudden and unexpected. Besides the injury itself, there are extensive, life-changing consequences $[1,2]$. The onset of an SCI is accompanied by a massive amount of stress. The psychosocial adaptation to disability has become an important part

Yanbo Wang

wang_yb@tongji.edu.cn

$\triangle$ Xudong Zhao zhaoxd@tongji.edu.cn

1 Department of Psychosomatic Medicine, Shanghai East Hospital, Tongji University School of Medicine, Shanghai, China

2 Department of Occupational and Social Rehabilitation, Sunshine Rehabilitation Center, Shanghai, China of a patient's rehabilitation [3, 4]. Some studies reveal that the SCI population has higher incidences of psychological morbidities when compared with the general population. As two systematic reviews reported, approximately $30 \%$ of individuals with SCI are at the risk of having a depressive disorder. About 19-26\% of the SCI population is diagnosed as suffering from depression [5, 6]. The prevalence of anxiety, as estimated from the individual's self-reported responses, ranges from 15 to $32 \%$ [7]. The traumatic SCI population has a significant 1.29-fold increased risk of anxiety or depression compared to the group with other health conditions [8]. Posttraumatic stress disorder (PTSD) is another morbidity reported and its prevalence varies between 7 and 44\% [9]. Depression, anxiety, and PTSD have become the three most common psychological morbidities in the SCI population. 
Although traumatic SCI is distressing and detrimental, survivors often also experience positive psychological consequences, such as resilience and posttraumatic growth (PTG) [10]. Resilience refers to the personal qualities that enable individuals to adapt to adversity [11]. People who demonstrated high levels of resilience were better at applying resources to protect themselves from suffering caused by the medical, psychological and social problems and ongoing daily demands as results of SCI [12]. PTG is defined as positive psychological change experienced after a struggle with challenging life circumstances [13]. A growing body of literature provides compelling evidence of PTG arising in the aftermath of an individual's traumatic experience [14-16]. PTG has attracted considerable attention over the past decade for its adaptive value. There are more than 1 million people in Mainland China currently living with an SCI, and approximately 120,000 new cases are reported every year [17]. SCI incidence is on the rise with the nations development and the increasing occurrence of natural disasters. China is still in the early stages of developing its disability rehabilitation programs. SCI treatment focuses on emergency life-saving measures and immediate physical problems. It largely ignores the psychological consequences and rehabilitation. Some health-care providers lack awareness of the humanistic care and psychological rehabilitation [18]. Since 2009, the Chinese Association of Persons with Physical Disability has been running a government-supported rehabilitation project called 'halfway home'. The purpose of the project is to provide comprehensive rehabilitation for the SCI population. It focuses on physical, mental, and social aspects. More than 500 individuals with SCI have participated in the 'halfway home' project since 2009. More and more people are aware of the importance of psychological rehabilitation.

The psychological outcomes of SCI have been well studied in western cultures. SCI occurs more in China as it develops, but psychological rehabilitation is lagging far behind. So, to improve the understanding of how people with SCI in China may suffer differently from their western counterparts, we will examine different psychological outcomes. The aims of this study were: (a) to explore the prevalence of the common psychological morbidities and positive psychological outcomes in the people with SCI in Mainland China, (b) to clarify the relationship between different psychological outcome variables, and (c) to identify the predictive effect of positive psychological outcomes on psychological morbidities. We also examined if demographic and injury-related variables could predict the onset of psychological morbidities. We hypothesized that low resilience and PTG would predict elevated rates of psychological morbidity.

\section{Methods}

\section{Participants and procedure}

The research proposal received ethical approval from the ethics committee of Tongji University and The Shanghai Sunshine Rehabilitation Center. Inclusion criteria included: (a) SCI caused by traumatic injury, (b) age between 18 and 65 years, and (c) at least 6 months after the injury occurred. Individuals with a known head injury or communication disorder were excluded from the study. The participants were recruited from the project of the 'halfway home' in Shanghai Sunshine Rehabilitation Center. The center provided comprehensive rehabilitation for individuals with SCI based on group intervention. A total of 320 people participated in the rehabilitation project between March 2014 and December 2016. Every participant was invited to complete the questionnaire before the group intervention started. The final study sample consisted of 300 participants who completed the questionnaires.

\section{Measures}

The participants provided demographic data (e.g., age, education, marital status, religion) and injury-related characteristics (e.g., cause of injury, length of time since their injury). The participants indicated the extent of environmental barriers on a 5-point Likert scale. From 1 for unrestricted, to 5 for extremely restricted.

\section{Psychological morbidities}

PTSD symptoms, anxiety, and depression were chosen as the indicators of psychological morbidity for the sample of SCI patients in Mainland China.

PTSD symptoms were assessed by the Impact of Event Scale-Revised (IES-R). It is a 22 -item self-report questionnaire designed to identify symptoms of PTSD [19, 20]. The degree of distress for each item is rated on a 5-point scale so the score ranges from 0 to 88 . Higher scores indicate a greater symptom load; 33 is generally used as the diagnostic cutoff in the literature [21]. It has been found to have good and stable psychometric properties [21]. Three core symptoms of PTSD, namely, re-experiencing, avoidance, and hyperarousal, are measured. The Chinese version of the IES-R has been found to have satisfactory psychometric properties and a score of 35 or greater was recommended as indicative of PTSD [22]. In the current study, Cronbach's $\alpha$ of PTSD symptoms was 0.95 and a score of 35 was used as a cutoff point.

Depression and anxiety were assessed by the Hospital Anxiety and Depression Scale (HADS). HADS is a selfreport measure comprised of 14 items with two 7 -item 
subscales; one for anxiety (HADS-A) and one for depression (HADS-D) [23]. It is a validated and reliable measure of mood states in people with SCI. Each item is scored on a 4-point Likert-type scale ranging from 0 (no problem) to 3 (high level of problems). The score for each subscale is obtained by simple summation of individual items with scores in each subscale, ranging from 0 to 21 . A score of seven or less indicates a non-anxiety/depression case, 8-10 a borderline case, and 11 or above a definite case [24]. In the Chinese version, 9 was considered the better clinically significant cutoff score [25].

\section{Positive psychological outcomes}

Positive psychological outcomes included measures of resilience and PTG.

Resilience was measured by the Connor-Davidson Resilience Scale (CD-RISC) [11], which has acceptable psychometric properties including test-retest reliability and construct validity. Items are scored on a Likert-type scale using a 5-point scale ranging from 0 (not true at all) to 4 (true nearly all the time). Participants rated their perceived resilience over the prior month. Higher scores are indicative of greater resilience [11]. The Chinese version of the CD-RISC [26] has shown good psychometric properties, with a Cronbach's $\alpha$ for the full scale of 0.91 . In the present study, Cronbach's $\alpha$ of CD-RISC was 0.90 .

PTG was measured by the Posttraumatic Growth Inventory (PTGI), which is a 21-item questionnaire with five subscales [13]. The PTGI has acceptable validity, internal consistency, and test-retest reliability over 2 months [13]. Participants responded on a 6-point scale, 0 for no change and 5 for complete change. Higher scores represent better levels of PTG [13]. The Chinese version of PTGI (PTGI-C) contained only 20 items as it did not include item 18 'I have a stronger religious faith'. The total possible score was 100 [27]. Some studies endorsed that a mean item score of PTGI $>3$ reached moderate and above levels of PTG $[28,29]$. The current study adopts the above criteria. The mean item score $>3$ and total score $>60$ is the cutoff point for moderate and above levels of PTG. For this study, the total PTGI-C internal consistency was 0.93.

\section{Statistical analysis}

The Statistical Package for Social Sciences (SPSS), version 16.0 (IBM SPSS Inc, Armonk, New York, USA), was used. The demographic and injury-related characteristics of the participants were tabulated. Descriptive analysis included the means and standard deviations of the main variables. We then conducted bivariate correlations to examine potential relations among demographic and injury-related and five psychological outcomes. This analysis was to identify potential covariates. The bivariate correlations were examined between the five psychological outcome variables. Hierarchical linear regressions were performed to determine how much the demographic and injury-related variables, resilience, and PTG explained the variance in the number of the three psychological morbidities. Only the factors that were found to be positively correlated were included in the regression. The 'enter' method was used for all steps. Cronbach's $\alpha$ coefficients were calculated for all scales. All statistical tests were two-tailed with an $\alpha$ level of 0.05 .

\section{Results}

\section{Participant characteristics}

The mean age of the sample was $45.81 \pm 12.89$ years (range, 18-65) and the mean age at injury was $34.11 \pm 14.35$ years (range, 3-68). Duration since injury was $12.06 \pm 13.26$ years on average. Demographic and injury-related characteristics are summarized in Table 1.

\section{Descriptive statistics and prevalence of psychological morbidities}

Table 2 shows means and standard deviations for measures of the PTSD symptoms, anxiety and depression, resilience, and PTG. Of 300 participants, the prevalence estimate for probable PTSD was 105 (35\%). This estimate came from the clinically significant cutoff score for PTSD of 35 . The prevalence estimates for probable anxiety and depression were 86 (29\%) and 81 (27\%), respectively. In Connor and Davidson's study [11], the mean resilience score for psychiatric outpatients was 68 . Their recommendation was that 68 be the cutoff between poor and acceptable resilience. The 68 score was also used to assess the level of resilience in people with SCI in this study [12, 30]. In this study, of the 300 participants, 96 (32\%) showed acceptable resilience, whereas 204 (68\%) showed poor resilience. Scores $>60$ were considered to indicate a moderate level of PTG, and the prevalence of moderate and above PTG in the sample was $51 \%(n=154)$.

\section{Correlation analysis}

Pearson correlation analyses were firstly used to examine the relationship between patients' demographic and injuryrelated variables and the different psychological variables (see Table 2). Of the potential covariates, age, age at injury, and extent of environmental barriers were positively correlated with PTSD, anxiety, and depression. Gender and marital status were negatively correlated with PTSD and anxiety. Duration since injury was negatively related with 
Table 1 Demographic and injury-related characteristics $(N=300)$

\begin{tabular}{|c|c|}
\hline Characteristics & $N(\%)$ \\
\hline \multicolumn{2}{|l|}{ Gender } \\
\hline Male & $180(60)$ \\
\hline Female & $120(40)$ \\
\hline \multicolumn{2}{|l|}{ Marital status } \\
\hline Unmarried & $158(53)$ \\
\hline Currently married & $111(37)$ \\
\hline Divorced & $31(10)$ \\
\hline \multicolumn{2}{|l|}{ Education } \\
\hline Junior high school and lower & $142(47)$ \\
\hline Senior high school & $106(35)$ \\
\hline University degree and above & $52(17)$ \\
\hline \multicolumn{2}{|l|}{ Religion } \\
\hline Yes & $50(17)$ \\
\hline No & $250(83)$ \\
\hline \multicolumn{2}{|l|}{ Duration since injury } \\
\hline$<1$ year post-injury & $73(24)$ \\
\hline $1-3$ years post-injury & 47 (16) \\
\hline$>3$ years post-injury & $65(22)$ \\
\hline$>10$ years post-injury & $115(38)$ \\
\hline \multicolumn{2}{|l|}{ Level and extent of lesion } \\
\hline Tetraplegia & $71(24)$ \\
\hline Paraplegia & $229(76)$ \\
\hline \multicolumn{2}{|l|}{ Completeness of lesion } \\
\hline Complete & $143(41)$ \\
\hline Incomplete & $157(52)$ \\
\hline \multicolumn{2}{|l|}{ Extent of environmental barriers } \\
\hline Less than 3 & $110(37)$ \\
\hline More and equal to 3 & $190(63)$ \\
\hline \multicolumn{2}{|l|}{ Employment } \\
\hline In paid workforce & $21(7)$ \\
\hline Not in paid workforce & $279(93)$ \\
\hline
\end{tabular}

anxiety and depression. This meant that the individuals who were male, single or divorced, and in the early stages of following the SCI were the highest risk group for developing one or more of the psychological morbidities. Religion and employment were positively associated with resilience and PTG. Additionally, duration since injury showed a positive relation to PTG.

As shown in Table 3, the relationships between five psychological outcome variables were examined using bivariate correlation analyses. Strong positive relationships between resilience and PTG $(r=0.57, p<0.01)$ as well as between PTSD, anxiety, and depression were found $(r=0.69,0.62$, and 0.71 , respectively). Negative correlations between resilience and PTSD, anxiety, and depression were found. Individuals with lower levels of resilience tended to report more symptoms of PTSD, anxiety, and depression. PTG was negatively correlated with PTSD and anxiety, suggesting that the individuals who achieved more PTG tended to report less negative outcomes, especially depression.

\section{Hierarchical regression analysis}

Hierarchical regression analysis was performed to identify the relative contribution of the independent variables of interest to the three psychological morbidities (see Table 4). The three common psychological morbidities were regressed separately as dependent variables. The independent variables were entered into the equation through three steps. All variables related to the three psychological morbidities were entered into the model. We used three steps because we wanted to enter the more stable variables as one block into the equation before entering the less stable variables (again, as one block).

Resilience can be considered as personal trait and more stable than PTG, so it was entered into the equation before
Table 2 Bivariate correlations amongst demographic and injury-related variables and psychological outcome $\operatorname{variables}(N=300)$

\begin{tabular}{llllll}
\hline Variable & Resilience & PTG & PTSD symptoms & Anxiety & Depression \\
\hline Age & 0.09 & 0.10 & $0.23^{* *}$ & $0.13^{*}$ & $0.14^{*}$ \\
Age at injury & -0.03 & -0.02 & $0.26^{* *}$ & $0.28^{* *}$ & $0.23^{* *}$ \\
Gender & 0.05 & -0.04 & $-0.13^{*}$ & $-0.15^{* *}$ & -0.07 \\
Marital status & -0.06 & 0.10 & $-0.14^{*}$ & $-0.14^{*}$ & -0.12 \\
Education & 0.11 & -0.02 & -0.13 & -0.09 & -0.07 \\
Religion & $0.18^{*}$ & $0.12^{*}$ & -0.04 & -0.00 & -0.07 \\
Duration since injury & 0.09 & $0.23^{* *}$ & 0.11 & $-0.24^{* *}$ & $-0.20^{* *}$ \\
Level and extent of lesion & 0.06 & -0.04 & -0.09 & -0.07 & -0.03 \\
Completeness of lesion & -0.09 & 0.03 & -0.03 & -0.09 & -0.09 \\
Extent of environmental barrier & $-0.32^{* *}$ & $-0.16^{*}$ & $0.28^{* *}$ & $0.25^{* *}$ & $0.28^{* *}$ \\
Employment & $0.19^{*}$ & $0.13^{*}$ & -0.03 & -0.03 & -0.07 \\
\hline
\end{tabular}

$P T G$ posttraumatic growth, $P T S D$ posttraumatic stress disorder. $* P<0.05, * * P<0.01$ 
PTG. The demographic and injury-related variables were entered in the first step. The second step was resilience, and PTG was the final step. Overall, different combinations of predictors accounted for $16 \%$ of the variance of PTSD results. They also accounted for $31 \%$ of the variance in anxiety and $32 \%$ of the variance in depression. In all the models, resilience had the greatest predictive effect on the dependent variable, and it accounted for $10 \%, 16 \%$, and $25 \%$ of the variance of PTSD, anxiety, and depression, respectively. Although PTG was negatively correlated with the three independent variables, it was not the significant predictor of the three psychological morbidities. 'Gender' and 'extent of environmental barriers' positively predicted PTSD, anxiety, and depression.

\section{Discussion}

This study examined the prevalence of three psychological morbidities (PTSD, anxiety, and depression) and two positive psychological outcomes (resilience and PTG) in the

Table 3 Bivariate correlations amongst resilience, PTG, PTSD, anxiety and depression $(N=300)$

\begin{tabular}{llllll}
\hline Variable & 1 & 2 & 3 & 4 & 5 \\
\hline 1. Resilience & 1 & & & & \\
2. PTG & $0.57^{* *}$ & 1 & & & \\
3. PTSD symptoms & $-0.34^{* *}$ & $-0.12^{*}$ & 1 & & \\
4. Anxiety & $-0.42^{* *}$ & $-0.27^{* *}$ & $0.69^{* *}$ & 1 & \\
5. Depression & $-0.51^{* *}$ & $-0.40^{* *}$ & $0.62^{* *}$ & $0.71^{* *}$ & 1 \\
$\quad$ Mean & 59.45 & 59.05 & 27.18 & 6.41 & 6.07 \\
SD & 18.03 & 18.07 & 17.52 & 4.15 & 4.30 \\
$\quad$ Range & $11-115$ & $0-95$ & $0-73$ & $0-19$ & $0-19$ \\
\hline
\end{tabular}

$P T G$ posttraumatic growth, $P T S D$ posttraumatic stress disorder. $* P<0.05, P<0.01$
SCI population. The probable PTSD prevalence estimate in our sample was $35 \%$, which is high compared with previous SCI studies reports [31, 32]. A systematic review reported that the prevalence of PTSD post-injury varied from 6 to $45 \%$. These ranges demonstrated an overall decreasing trend over time [33]. However, in this study, even for the individuals who had acquired their injury 10 or more years prior to the study, the rates reached $33 \%$. This is almost no less than the overall total. This result can be understood with the following context. Firstly, individuals with traumatic SCI are more susceptible to PTSD, experiencing an SCI increases the incidence of PTSD development relative to a control group of people with other health conditions (7\%) [34]. Secondly, China is a developing country whose disability rehabilitation is in its infancy. Medical care for people with SCI is focused on emergency life-saving measures and the physical problems. However, it ignores their psychological problems and rehabilitation. As one qualitative study revealed, most individuals with SCI have experienced a series of traumas, including disabled body, a broken marriage, distress from complications, and isolation from society [18]. Thirdly, specialized rehabilitation institutions and multidisciplinary establishments have been inadequate in the field of SCI rehabilitation in China. Most new SCI patients have had to discharge from acute rehabilitation before they had the functional skills necessary to successfully return to the community [18].

The prevalence of anxiety in this study was $29 \%$, which is consistent with the anxiety levels reported by hospitals (27\%) and communities (29\%) in a prior meta-analysis [7]. The proportion of depression in this study was $27 \%$, which is consistent with the systematic review of Craig et al. [5] but higher than the meta-analysis by Williams and Murray (varying from 19 to 26\%) [6]. These findings support the observation that people with SCI from both Eastern and Western backgrounds have an increased risk of suffering psychological morbidities.

Table 4 Hierarchical regression analysis for predictors of PTSD symptoms, anxiety, and depression $(N=300)$

\begin{tabular}{|c|c|c|c|c|c|c|c|c|c|c|c|c|}
\hline \multirow[t]{2}{*}{ Variables } & \multicolumn{4}{|c|}{ PTSD symptoms } & \multicolumn{4}{|c|}{ Anxiety } & \multicolumn{4}{|c|}{ Depression } \\
\hline & $\bar{B}$ & $\beta$ & $p$ & $R^{2}\left(\Delta R^{2}\right)$ & $\bar{B}$ & $\beta$ & $p$ & $R^{2}\left(\Delta R^{2}\right)$ & $\bar{B}$ & $\beta$ & $p$ & $R^{2}\left(\Delta R^{2}\right)$ \\
\hline \multicolumn{4}{|c|}{ Step 1 Demographic and injured variable } & $13.9 \%$ & & & & $18.4 \%$ & & & & $12.3 \%$ \\
\hline Gender & -6.59 & -0.19 & 0.04 & & -2.71 & -0.31 & 0.00 & & -1.47 & -0.16 & 0.07 & \\
\hline Age & 0.14 & 0.12 & 0.51 & & -0.02 & -0.05 & 0.77 & & 0.06 & 0.17 & 0.28 & \\
\hline Age at injury & -0.05 & -0.05 & 0.78 & & 0.03 & 0.11 & 0.51 & & -0.02 & -0.07 & 0.69 & \\
\hline Marital & -0.29 & -0.01 & 0.90 & & -0.32 & -0.05 & 0.59 & & -0.10 & -0.02 & 0.88 & \\
\hline Duration since injury & 0.08 & 0.01 & 0.96 & & -0.04 & -0.01 & 0.93 & & -0.36 & -0.11 & 0.45 & \\
\hline Environmental barriers & 5.14 & 0.32 & 0.00 & & 0.87 & 0.21 & 0.02 & & 1.20 & 0.28 & 0.00 & \\
\hline Step 2 Resilience & -0.25 & -0.27 & 0.00 & $20.5 \%(6.6 \%)$ & -0.09 & -0.38 & 0.00 & $31.1 \%(12.7 \%)$ & -0.12 & -0.48 & 0.00 & $32.7 \%(20.3 \%)$ \\
\hline Step 3 PTG & -0.04 & -0.04 & 0.72 & $20.6 \%(0.1 \%)$ & -0.03 & -0.10 & 0.28 & $31.8 \%(0.7 \%)$ & -0.02 & -0.08 & 0.38 & $33.1 \%(0.4 \%)$ \\
\hline
\end{tabular}

$P T G$ posttraumatic growth, $P T S D$ posttraumatic stress disorder 
As the common positive psychological outcomes, resilience and PTG were explored in the present study. The participants reported lower levels of resilience but better levels of PTG than other prior reports. A prior study about SCI in America found that 58\% of 60 respondents reported moderate to high levels of resilience [30]. Another prior study in Australia showed that $60-70 \%$ of people with SCI reported satisfactory levels of resilience [12]. Only $32 \%$ of the participants reported satisfactory resilience in the current study. As for PTG, our findings showed that $51 \%$ reported moderate to high levels of PTG, which was lower than the $79 \%$ of reporters in a prior study [16]. But $99 \%$ of respondents recognized that at least one positive change had occurred in their life as a result of their SCI, which was consistent with the results of January et al.'s study [35]. One possible explanation for the difference in prevalences of the resilience might be the social and cultural differences between different countries. Most people with SCI in Mainland China came from low-income families. The limited social welfare and difficult employment status in our country also made their burdens heavier.

The relationships between the demographic and injuryrelated variables and the five psychological outcomes were examined. Consistent with the existing studies [8, 32, 36], factors including age, age at injury, gender, and marital status were all shown to be related to psychological morbidities. The findings showed that older people, males, single or divorced individuals, and patients in the early stage of injury were most susceptible to the psychological disorders. Self-reporting about environmental barriers reflected the individual's perception of their social participation ability. It positively correlated with the three psychological morbidities. This finding supports the view in prior studies that the negative trauma-related self-appraisals are related to psychopathology [32]. Additionally, individuals who were religious and had employment experience following the SCI showed better resilience and PTG.

As expected, the three psychological morbidities showed strong associations with each other. Further analysis found that $12 \%$ of participants' scores in PTSD, anxiety, and depression all exceeded the clinically significant cutoff score. A strong correlation between resilience and PTG was found, which was consistent with previous studies [37]. This revealed that personalities with high resilience could contribute to the PTG of people with SCI. The study found strong negative associations between the three morbidities and the two positive outcomes.

Hierarchical regression analysis was used to analyze the predictors of PTSD symptoms, anxiety, and depression. 'Male gender' predicted change in PTSD symptoms and anxiety, which means the male patients are at high risk for PTSD and anxiety. 'The extent of environmental barriers' also predicted change in PTSD, anxiety, and depression.
Training people how to address the environmental barriers and improve their community reintegration might reduce the prevalence of morbidities.

Resilience was a significant negative predictor of development of PTSD, anxiety, and depression. The findings added to the evidence that high levels of resilience can predict decline in the incidences of psychological morbidities [37]. Demonstrations of resilience might work as a buffer against the distress caused by the consequences of SCI [12]. Although PTG was found to be correlated with the three morbidities, it was not a strong predictor. Some studies reveal that people with high levels of PTG will recover more, and others conclude that PTG always coexists with mental distress [15, 18, 38, 39]. The rehabilitation mechanism will be a good subject for further study.

\section{Clinical implication}

The findings of this study have several important clinical implications. First, there are high levels of PTSD, anxiety, and depression in the SCI population in Mainland China. They should be identified early in the rehabilitation process. Many people could benefit from psychological interventions against the psychological morbidities. Second, the negative correlations between positive outcomes and morbidities suggest that the incorporation of positive psychology techniques, such as focusing on finding resilience resources and promoting positive growth, into the psychological intervention would likely prove beneficial for the SCI population. Additionally, resilience is the primary defense against PTSD, anxiety, and depression, but the participants' resilience in the study is still at lower levels compared with prior studies. Enhancing the resilience level of people with SCI may be effective in preventing the occurrences of morbidities. Last, the predictive effect of the extent of environmental barriers can aid clinical rehabilitation workers. They should make a detailed assessment the individual's perception of their environmental barriers and provide targeted training.

\section{Limitation and further study}

There are several limitations to this study. The findings can only be generalized to individuals who have experienced traumatic injuries. Recruitment of the sample was from only one rehabilitation center, and the percentages are only estimates of the three morbidities. These estimates resulted from screening of the patients' self-report. Applying the results to a larger population will require caution due to representative issues in the study group. The duration since the injury is an important factor, but not studied as much as it is necessary here. Larger, longitudinal studies, including patients at all stages of injury recovery, are needed. With 
that we can understand the prevalence and developmental trajectory of different outcomes in the SCI population. As reported in previous studies [9], many factors affected the prevalence of psychological morbidities. It is necessary to explore the risk factors for PTSD, anxiety, and depression in a larger sample. Correlation analysis showed that both resilience and PTG had negative correlations with the three psychological morbidies. Yet, resilience showed a predictive effect for them while PTG did not. Further research is needed to explain the mechanism of positive psychological outcomes on these morbidities.

Acknowledgements We thank all the persons who participated in this study and the Shanghai Sunshine Hospital. This work was supported by the project of the Shanghai Disabled Person's Federation (K2016027).

\section{Compliance with ethical standards}

Conflict of interest The authors declare that they have no conflict of interest.

\section{References}

1. Devivo M. Epidemiology of traumatic spinal cord injury: trends and future implications. Spinal Cord. 2012;50:365-72.

2. World Health Organization. International Perspectives on Spinal Cord Injury. Geneva: World Health Organization; 2013.

3. Dickson A, Ward R, O'Brien G, Allan D, O'Carroll R. Difficulties adjusting to post-discharge life following a spinal cord injury: an interpretative phenomenological analysis. Psychol Health Med. 2011;16:463-74.

4. Byrnes M, Beilby J, Ray P, McLennan R, Ker J, Schug S. Patientfocused goal planning process and outcome after spinal cord injury rehabilitation: quantitative and qualitative audit. Clin Rehabil. 2012;26:1141-9.

5. Craig A, Tran Y, Middleton J. Psychological morbidity and spinal cord injury: a systematic review. Spinal Cord. 2009;47:108-14.

6. Williams R, Murray A. Prevalence of depression after spinal cord injury: a meta-analysis. Arch Phys Med Rehabil. 2015;96:133-40.

7. Le J, Dorstyn D. Anxiety prevalence following spinal cord injury: a meta-analysis. Spinal Cord. 2016;54:570-8.

8. Lim SW, Shiue YL, Ho CH, Yu SC, Kao PH, Wang JJ, et al. Anxiety and depression in patients with traumatic spinal cord injury: a nationwide population-based cohort study. PLoS ONE. 2017;12:14

9. Otis C, Marchand A, Courtois F. Risk factors for posttraumatic stress disorder in persons with spinal cord injury. Top Spinal Cord Inj Rehabil. 2012;18:253-63.

10. Quale AJ, Schanke AK. Resilience in the face of coping with a severe physical injury: a study of trajectories of adjustment in a rehabilitation setting. Rehabil Psychol. 2010;55:12-22.

11. Connor KM, Davidson JR. Development of a new resilience scale: the Connor-Davidson Resilience Scale (CD-RISC). Depress Anxiety. 2003;18:76-82.

12. Guest R, Craig A, Tran Y, Middleton J. Factors predicting resilience in people with spinal cord injury during transition from inpatient rehabilitation to the community. Spinal Cord. 2015;53:682-6.
13. Tedeschi RG, Calhoun LG. The posttraumatic growth inventory: measuring the positive legacy of trauma. J Trauma Stress. 1996;9:455-71.

14. Chun S, Lee Y. The experience of posttraumatic growth for people with spinal cord injury. Qual Health Res. 2008;18:877-90.

15. Griffiths HC, Clinpsy D, Kennedy P. Continuing with life as normal: positive psychological outcomes following spinal cord injury. Top Spinal Cord Inj Rehabil. 2012;18:241-52.

16. Kalpakjian CZ, McCullumsmith CB, Fann JR, Richards JS, Stoelb $\mathrm{BL}$, Heinemann AW, et al. Post-traumatic growth following spinal cord injury. J Spinal Cord Med. 2014;37:218-25.

17. Feng Y, Zhu H, Liu Y. Treatment of spinal cord Injury. Chin J Neurosurg Dis Res. 2008;7:279-80.

18. Wang Y, Wang H, Wang Z, Xie H, Shi J, Zhao X. The process of posttraumatic growth in individuals with traumatic spinal cord injury in Mainland China: An interpretative phenomenological analysis. J Health Psychol. 2017;22:637-49.

19. Weiss DS, Marmar CR. The impact of event scale-Revised. In: Wilson JP, Keane TM, editors. Assessing psychological trauma and PTSD. New York: Guilford Press; 1997. p. 399-411.

20. Weiss DS, Marmar CR. The impact of event scale-Revised. In: Wilson JP, Keane TM, editors. Assessing psychological trauma and PTSD. New York: Guilford Press; 2004. p. 168-89.

21. Creamer M, Bell R, Failla S. Psychometric properties of the Impact of Event Scale-Revised. Behav Res Ther. 2003;41:1489-96.

22. Huang G, Zhang Y, Xiang H. The Chinese version of the impact of event scale-revised: reliability and validity. Chin Ment Health J. 2006;20:28-32.

23. Zigmond A, Snaith R. The hospital anxiety and depression scale. Acta Psychiatr Scand. 1983;67:361e70.

24. Arving C, Glimelius B, Brandberg Y. Four weeks of daily assessments of anxiety, depression and activity compared to a point assessment with the Hospital Anxiety and Depression Scale. Qual Life Res. 2008;17:95e104.

25. Zheng L, Wu Y, Li H. Application of Hospital Anxiety and Depression Scale in general hospital: an analysis in reliability and validity. Shanghai Arch Psychiatry. 2003;15:264-6.

26. Yu X, Zhang J. Factor analysis and psychometric evaluation of The Connor-Davidson Resilience Scale(CD-RISC) with Chinese people. Soc Behav Personal. 2007;35:19-30.

27. Wang Y, Wang H, Wang J, Wu J, Liu X. Prevalence and predictors of posttraumatic growth in accidentally injured patients. $\mathrm{J}$ Clin Psychol Med Settings. 2013;20:3-12.

28. Tang CS. Positive and negative postdisaster psychological adjustment among adult survivors of the Southeast Asian earthquake-tsunami. J Psychosom Res. 2006;61:699-705.

29. Schroevers MJ, Teo I. The report of posttraumatic growth in Malaysian cancer patients: relationships with psychological distress and coping strategies. Psychooncology. 2008;17:1239-46.

30. Kilic SA, Dorstyn DS, Guiver NG. Examining factors that contribute to the process of resilience following spinal cord injury. Spinal Cord. 2013;51:553-7.

31. Krause JS, Carter R, Zhai YS, Reed K. Psychologic factors and risk of mortality after spinal cord injury. Arch Phys Med Rehab. 2009;90:628-33.

32. Schonenberg M, Reimitz M, Jusyte A, Maier D, Badke A, Hautzinger M. Depression, posttraumatic stress, and risk factors following spinal cord injury. Int J Behav Med. 2014;21:169-76.

33. Heron-Delaney M, Kenardy J, Charlton E, Matsuoka Y. A systematic review of predictors of posttraumatic stress disorder (PTSD) for adult road traffic crash survivors. Injury. 2013;44:1413-22. 
34. Kessler RC, Berglund P, Demler O, Jin R, Merikangas KR. Lifetime prevalence and age of onset distributions of DSM-IV disorders in the National Comorbidity Survey Replication. Arch Gen Psychiatry. 2005;62:593-602.

35. January AM, Zebrack K, Chlan KM. Understanding post-traumatic growth following pediatric-onset spinal cord injury: the critical role of coping strategies for facilitating positive psychological outcomes. Dev Med Child Neurol. 2015;11:1143-9.

36. Pollock K, Dorstyn D, Butt L, Prentice S. Posttraumatic stress following spinal cord injury: a systematic review of risk and vulnerability factors. Spinal Cord. 2017;55:800-811.
37. Ying L, Wang Y, Lin C, Chen C. Trait resilience moderates the longitudinal linkage between adolescent posttraumatic stress disorder symptoms and posttraumatic growth. Sch Psychol Int. 2016;37:207-22.

38. Min JA, Lee CU, Hwang SI, Shin JI, Lee BS, Han SH, et al. The moderation of resilience on the negative effect of pain on depression and post-traumatic growth in individuals with spinal cord injury. Disabil Rehabil. 2014;36:1196-202.

39. Bonanno GA, Kennedy P, Galatzer-Levy IR, Lude P, Elfstrom ML. Trajectories of resilience, depression, and anxiety following spinal cord injury. Rehabil Psychol. 2012;57:236-47. 